\title{
Author Index Volume 34 (2013)
}

The issue number is given in front of the pagination

Abbott,A.C., C.C. Toledo, F.C.Aranguiz, N.C.Inestrosa and L. Varela-Nallar, Tetrahydrohyperforin Increases Adult Hippocampal Neurogenesis in Wild-Type and APPswe/PS1 1 E9 Mice (4) 873885

Aicardi, G., see Balietti, M. (4) 887-896

Aldudo, J., see Recuero, M. (3) 637-647

Alegret, M., see Espinosa, A. (3) 769-780

Alia, A., see van Duijn, S. (4) 1051-1059

Ames, D., see Ellis, K.A. (4) 861-871

Ames, D., see Rembach, A. (1) 171-182

Amodio, P., see Babiloni, C. (3) 707-725

Ancelin, M.-L., E. Ripoche, A.-M. Dupuy, P. Barberger-Gateau, S. Auriacombe, O. Rouaud, C. Berr, I. Carrière and K. Ritchie, Sex Differences in the Associations Between Lipid Levels and Incident Dementia (2) 519-528

Ancelin, M.-L., see Tierney, M.C. (3) 601-608

Andreasen, N., see Olsson, B. (3) 673-679

Annamalai, B., see Won, J.-S. (3) 621-635

Annas, P., see Olsson, B. (3) 673-679

Aranguiz, F.C., see Abbott, A.C. (4) 873-885

Asif, A.R., see Dieks, J.-K. (2) 387-397

Auriacombe, S., see Ancelin, M.-L. (2) 519-528

Babiloni, C., F. Vecchio, C.D. Percio, S. Montagnese, S. Schiff, R. Lizio, G. Chini, G. Serviddio, N. Marzano, A. Soricelli, G.B. Frisoni, P.M. Rossini and P. Amodio, Resting State Cortical Electroencephalographic Rhythms in Covert Hepatic Encephalopathy and Alzheimer's Disease (3) 707-725

Babiloni, C., R. Lizio, C.D. Percio, N. Marzano, A. Soricelli, E. Salvatore, R. Ferri, F.I.I. Cosentino, G. Tedeschi, P. Montella, S. Marino, S.D. Salvo, G. Rodriguez, F. Nobili, F. Vernieri, F. Ursini, C. Mundi, J.C. Richardson, G.B. Frisoni and P.M. Rossini, Cortical Sources of Resting State EEG Rhythms are Sensitive to the Progression of Early Stage Alzheimer's Disease (4) 1015-1035

Backus, C., see Kim, B. (3) 727-739

Bahri, S., see Chien, D.T. (2) 457-468
Balietti, M., B. Giorgetti, T. Casoli, M. Solazzi, F. Tamagnini, C. Burattini, G. Aicardi and P. Fattoretti, Early Selective Vulnerability of Synapses and Synaptic Mitochondria in the Hippocampal CA1 Region of the Tg2576 Mouse Model of Alzheimer's Disease (4) 887-896

Bannach, O., see Wang-Dietrich, L. (4) 985-994

Barber, R., see O'Bryant, S.E. (4) 841-849

Barber, R., see Pavlik, V. (2) 449-456

Barberger-Gateau, P., see Ancelin, M.-L. (2) 519-528

Barnes, J., J.W. Bartlett, N.C. Fox, J.M. Schott and for the Alzheimer's Disease Neuroimaging Initiative, Targeted Recruitment Using Cerebrospinal Fluid Biomarkers: Implications for Alzheimer's Disease Therapeutic Trials (2) 431-437

Bartlett, J.W., see Barnes, J. (2) 431-437

Bartnik, D., see van Groen, T. (3) 609-620

Bassi, V., see Pimouguet, C. (1) 307-314

Basun, H., see Olsson, B. (3) 673-679

Batt, M., see Jonveaux, T.R. (1) 325-338

Beach, T.G., see Dugger, B.N. (2) 529-536

Benetos, A., see Jonveaux, T.R. (1) 325-338

Benito-León, J., see Moreno-Ramos, T. (3) 659-664

Berg, A.I., see Rolstad, S. (4) 949-956

Berg, D., see Laske, C. (2) 367-375

Berger, E., see Le Ber, I. (2) 485-499

Berger, K.L., see Zhu, D.C. (4) 969-984

Berman, A., see Blautzik, J. (3) 741-754

Bermejo-Pareja, F., see Carmona, P. (4) 911-920

Bermejo-Pareja, F., see Moreno-Ramos, T. (3) 659-664

Berr, C., see Ancelin, M.-L. (2) 519-528

Berres, M., see Schmid, N.S. (2) 537-546

Besmehn, A., see Wang-Dietrich, L. (4) 985-994

Birkmann, E., see Wang-Dietrich, L. (4) 985-994

Bjerke, M., see Rolstad, S. (4) 949-956

Blanc, F., see Le Ber, I. (2) 485-499

Blautzik, J., D. Keeser, A. Berman, M. Paolini, V. Kirsch, S. Mueller, U. Coates, M. Reiser, S.J. Teipel and T. Meindl, Long-Term Test-Retest Reliability of Resting-State Networks in Healthy Elderly Subjects and Patients with Amnestic Mild Cognitive Impairment (3) 741-754 
Blazquez-Llorca, L., Á. Merchán-Pérez, J.-R. Rodríguez, J. Gascón and J. DeFelipe, FIB/ SEM Technology and Alzheimer's Disease: Three-Dimensional Analysis of Human Cortical Synapses (4) 995-1013

Blennow, K., see Olsson, B. (3) 673-679

Blondiaux, E., see Désiré, L. (2) 469-483

Boada, M., see Espinosa, A. (3) 769-780

Boeve, B.F., see Geda, Y.E. (2) 501-507

Bolognin, S., see Valenti, M.T. (1) 263-271

Bombois, S., see Gabelle, A. (1) 297-305

Bondi, M.W., see Wierenga, C.E. (4) 921-935

Bonetto, N., see Tagliapietra, M. (1) 231-238

Bouvel, B., see Jonveaux, T.R. (1) 325-338

Bozoki, A.C., see Zhu, D.C. (4) 969-984

Brady, C.I., see Sillerud, L.O. (2) 349-365

Brice, A., see Le Ber, I. (2) 485-499

Brown, D.C., see Sillerud, L.O. (2) 349-365

Buée, L., see Gabelle, A. (1) 297-305

Bullido, M.J., see Recuero, M. (3) 637-647

Burattini, C., see Balietti, M. (4) 887-896

Bush, A.I., see Ellis, K.A. (4) 861-871

Bush, A.I., see Rembach, A. (1) 171-182

Cagnin, A., A. Leon, D. Vianello, D. Colavito, S. Favaretto, G. Zarantonello, A. Stecca, M. Ermani and A. Zambon, LDL Density and Oxidation are Modulated by PON1 Promoter Genotype in Patients with Alzheimer's Disease (2) 377-385

Cagnin, A., see Tagliapietra, M. (1) 231-238

Calabrese, P., see Kalbe, E. (4) 813-834

Caldeira, G.L., I.L. Ferreira and A.C. Rego, Impaired Transcription in Alzheimer's Disease: Key Role in Mitochondrial Dysfunction and Oxidative Stress (1) 115-131

Calero, M., see Carmona, P. (4) 911-920

Camu, W., see Le Ber, I. (2) 485-499

Camuzat, A., see Le Ber, I. (2) 485-499

Cannavo, A., see Femminella, G.D. (2) 341-347

Carbonare, L.D., see Valenti, M.T. (1) 263-271

Carbone, I., see Porcellini, E. (2) 439-447

Cardoso, S., see Silva, D. (3) 681-689

Carmona, P., M. Molina, M. Calero, F. BermejoPareja, P. Martínez-Martín and A. Toledano, Discrimination Analysis of Blood Plasma Associated with Alzheimer's Disease Using Vibrational Spectroscopy (4) 911-920

Carrière, I., see Ancelin, M.-L. (2) 519-528

Carrière, J., see Désiré, L. (2) 469-483

Casabona, D., see Montañés, M. (1) 133-137

Casadio, R., see Porcellini, E. (2) 439-447
Casoli, T., see Balietti, M. (4) 887-896

Chamberlain, R., see Sillerud, L.O. (2) 349-365

Chan, M., see Chong, M.S. (2) 399-405

Chatzistavraki, M., E. Kyratzi, A. Fotinopoulou, P. Papazafiri and S. Efthimiopoulos, Downregulation of A $\beta$ PP Enhances Both Calcium Content of Endoplasmic Reticulum and Acidic Stores and the Dynamics of Store Operated Calcium Channel Activity (2) 407-415

Chen, G., see Chong, M.S. (2) 399-405

Chiarolanza, G., see Dugger, B.N. (2) 529-536

Chien, D.T., S. Bahri, A.K. Szardenings, J.C. Walsh, F. Mu, M.-Y. Su, W.R. Shankle, A. Elizarov and H.C. Kolb, Early Clinical PET Imaging Results with the Novel PHF-Tau Radioligand [F-18]-T807 (2) 457-468

Chini, G., see Babiloni, C. (3) 707-725

Choi, J.H.K., G. Kaur, M.J. Mazzella, J. MoralesCorraliza, E. Levy and P.M. Mathews, Early Endosomal Abnormalities and Cholinergic Neuron Degeneration in Amyloid- $\beta$ Protein Precursor Transgenic Mice (3) 691-700

Chong, M.S., L.K. Goh, W.S. Lim, M. Chan, L. Tay, G. Chen, L. Feng, T.P. Ng, C.H. Tan and T.S. Lee, Gene Expression Profiling of Peripheral Blood Leukocytes Shows Consistent Longitudinal Downregulation of TOMM40 and Upregulation of KIR2DL5A, PLOD1, and SLC2A8 Among Fast Progressors in Early Alzheimer's Disease (2) 399-405

Chong, M.S., see Feng, L. (3) 649-657

Christianson, T.J.H., see Geda, Y.E. (2) 501-507

Chuzeville, S.B., see Jonveaux, T.R. (1) 325-338

Cinar, Y., see Wang-Dietrich, L. (4) 985-994

Clark, L.R., see Wierenga, C.E. (4) 921-935

Coates, U., see Blautzik, J. (3) 741-754

Colavito, D., see Cagnin, A. (2) 377-385

Collinson, S.L., see Feng, L. (3) 649-657

Cosentino, F.I.I., see Babiloni, C. (4) 1015-1035

Costa, M.S., see Espinosa, J. (2) 509-518

Couratier, P., see Le Ber, I. (2) 485-499

Cui, J., see Li, R. (3) 589-594

Cunha, R.A., see Espinosa, J. (2) 509-518

Daffner, K.R., see Perez, D.L. (1) 139-144

Dai, J., see Zhou, Y. (4) 937-947

Dal-Ré, R., K. Fauria, N. Gramunt and J.L. Molinuevo, Clinical Observational Research on Alzheimer's Disease: What Clinical Trial Registries Can Tell (1) 183-190

Dams-O’Connor, K., see Zhao, W. (2) 417-429 
Darby, D., see Ellis, K.A. (4) 861-871

Darcourt, J., see Fontaine, D. (1) 315-323

Dartigues, J.F., see Pimouguet, C. (1) 307-314

de Lucia, C., see Femminella, G.D. (2) 341-347

de Mendonça, A., see Silva, D. (3) 681-689

de Ronde, F.M., see Nabuurs, R.J.A. (4) 1037-1049

Dean, R.A., see Lachno, D.R. (4) 897-910

DeFelipe, J., see Blazquez-Llorca, L. (4) 995-1013

Defrancesco, M., J. Marksteiner, E. Deisenhammer, G. Kemmler, T. Djurdjevic and M. Schocke, Impact of White Matter Lesions and Cognitive Deficits on Conversion from Mild Cognitive Impairment to Alzheimer's Disease (3) 665-672

Deisenhammer, E., see Defrancesco, M. (3) 665-672

Demarche, L., see Jonveaux, T.R. (1) 325-338

DeMattos, R.B., see Lachno, D.R. (4) 897-910

Deramecourt, V., see Le Ber, I. (2) 485-499

Désiré, L., E. Blondiaux, J. Carrière, R. Haddad, O. Sol, P. Fehlbaum-Beurdeley, R. Einstein, W. Zhou and M.P. Pando, Blood Transcriptomic Biomarkers of Alzheimer's Disease Patients Treated with EHT 0202 (2) 469-483

Deudon, A., see Fontaine, D. (1) 315-323

Dev, S.I., see Wierenga, C.E. (4) 921-935

Devanand, D.P., see Zahodne, L.B. (4) 851-860

Devous, M., see O’Bryant, S.E. (4) 841-849

Devous, M.D., see O’Bryant, S.E. (3) 701-706

Dickerson, B.C., see Perez, D.L. (1) 139-144

Didic, M., see Le Ber, I. (2) 485-499

Dieks, J.-K., J. Gawinecka, A.R. Asif, D. Varges, K. Gmitterova, J.-H. Streich, H. Dihazi, U. Heinemann and I. Zerr, Low-Abundant Cerebrospinal Fluid Proteome Alterations in Dementia with Lewy Bodies (2) 387-397

Dietzsch, J., see Laske, C. (2) 367-375

Dihazi, H., see Dieks, J.-K. (2) 387-397

Dijkstra, J., see Nabuurs, R.J.A. (4) 1037-1049

Djurdjevic, T., see Defrancesco, M. (3) 665-672

Dodge, H.H., see Katsumata, Y. (1) 273-279

Doecke, J.D., see Rembach, A. (1) 171-182

Donatelli, L., see Valenti, M.T. (1) 263-271

Doody, R., see Pavlik, V. (2) 449-456

Dubois, B., see Le Ber, I. (2) 485-499

Dugger, B.N., J.A. Hidalgo, G. Chiarolanza, M. Mariner, J. Henry-Watson, L.I. Sue and T.G. Beach, The Distribution of Phosphorylated Tau in Spinal Cords of Alzheimer's Disease and NonDemented Individuals (2) 529-536

Dumurgier, J., see Gabelle, A. (1) 297-305

Dupuy, A.-M., see Ancelin, M.-L. (2) 519-528

Duyckaerts, C., see Le Ber, I. (2) 485-499
Dwyer, J., see Dwyer, R. (1) 145-154

Dwyer, R., O.A. Skrobot, J. Dwyer, M. Munafo and P.G. Kehoe, Using Alzgene-Like Approaches to Investigate Susceptibility Genes for Vascular Cognitive Impairment (1) 145-154

Edwards, M., see O’Bryant, S.E. (3) 701-706

Edwards, M., see O’Bryant, S.E. (4) 841-849

Efthimiopoulos, S., see Chatzistavraki, M. (2) 407-415

Einstein, R., see Désiré, L. (2) 469-483

Elizarov, A., see Chien, D.T. (2) 457-468

Ellis, K.A., see Rembach, A. (1) 171-182

Ellis, K.A., Y.Y. Lim, K. Harrington, D. Ames, A.I. Bush, D. Darby, R.N. Martins, C.L. Masters, C.C. Rowe, G. Savage, C. Szoeke, V.L. Villemagne, P. Maruff and the AIBL Research Group, Decline in Cognitive Function over 18 Months in Healthy Older Adults with High Amyloid- $\beta$ (4) 861-871

Ermani, M., see Cagnin, A. (2) 377-385

Ermani, M., see Tagliapietra, M. (1) 231-238

Espinosa, A., M. Alegret, S. Valero, G. Vinyes-Junqué, I. Hernández, A. Mauleón, M. Rosende-Roca, A. Ruiz, O. López, L. Tárraga and M. Boada, A Longitudinal Follow-Up of 550 Mild Cognitive Impairment Patients: Evidence for Large Conversion to Dementia Rates and Detection of Major Risk Factors Involved (3) 769-780

Espinosa, J., A. Rocha, F. Nunes, M.S. Costa, V. Schein, V. Kazlauckas, E. Kalinine, D.O. Souza, R.A. Cunha and L.O. Porciúncula, Caffeine Consumption Prevents Memory Impairment, Neuronal Damage, and Adenosine $\mathrm{A}_{2 \mathrm{~A}}$ Receptors Upregulation in the Hippocampus of a Rat Model of Sporadic Dementia (2) 509-518

Evert, B.A., see Lachno, D.R. (4) 897-910

Fallgatter, A.J., see Laske, C. (2) 367-375

Fandos, N., see Izco, M. (4) 835-839

Fattoretti, P., see Balietti, M. (4) 887-896

Fauria, K., see Dal-Ré, R. (1) 183-190

Faux, N.G., see Rembach, A. (1) 171-182

Favaretto, S., see Cagnin, A. (2) 377-385

Fehlbaum-Beurdeley, P., see Désiré, L. (2) 469-483

Feldman, E.L., see Kim, B. (3) 727-739

Femminella, G.D., G. Rengo, G. Pagano, C. de Lucia, K. Komici, V. Parisi, A. Cannavo, D. Liccardo, C. Vigorito, P.P. Filardi, N. Ferrara and D. Leosco, $\beta$-Adrenergic Receptors and $G$ Protein-Coupled Receptor Kinase-2 in Alzheimer's Disease: A New Paradigm for Prognosis and Therapy? (2) 341-347 
Feng, L., M.S. Chong, W.S. Lim, T.S. Lee, S.L. Collinson, P. Yap and T.P. Ng, Metabolic Syndrome and Amnestic Mild Cognitive Impairment: Singapore Longitudinal Ageing Study-2 Findings (3) 649-657

Feng, L., see Chong, M.S. (2) 399-405

Fengler, S., see Kalbe, E. (4) 813-834

Ferrara, N., see Femminella, G.D. (2) 341-347

Ferreira, I.L., see Caldeira, G.L. (1) 115-131

Ferri, R., see Babiloni, C. (4) 1015-1035

Fescharek, R., see Jonveaux, T.R. (1) 325-338

Feuerstein, G.G.Z., see Nyborg, A.C. (4) 957-967

Fiala, M., see Mizwicki, M.T. (1) 155-170

Figueiredo, L.S., see Schröder, N. (4) 797-812

Filardi, P.P., see Femminella, G.D. (2) 341-347

Fiorini, M., see Tagliapietra, M. (1) 231-238

Fleury, M., see Le Ber, I. (2) 485-499

Foldi, N.S., see Schmid, N.S. (2) 537-546

Fontaine, D., A. Deudon, J.J. Lemaire, M. Razzouk, P. Viau, J. Darcourt and P. Robert, Symptomatic Treatment of Memory Decline in Alzheimer's Disease by Deep Brain Stimulation: A Feasibility Study (1) 315-323

Fotinopoulou, A., see Chatzistavraki, M. (2) 407-415

Fowler, C.J., see Rembach, A. (1) 171-182

Fox, N.C., see Barnes, J. (2) 431-437

Freire, D., see Zhao, W. (2) 417-429

Frisoni, G.B., see Babiloni, C. (3) 707-725

Frisoni, G.B., see Babiloni, C. (4) 1015-1035

Froestl, W., A. Pfeifer and A. Muhs, Cognitive Enhancers (Nootropics). Part 3: Drugs Interacting with Targets other than Receptors or Enzymes. Disease-modifying Drugs (1) 1-114

Funke, S.A., see van Groen, T. (3) 609-620

Funke, S.A., see Wang-Dietrich, L. (4) 985-994

Gabelle, A., J. Dumurgier, O. Vercruysse, C. Paquet, S. Bombois, J.-L. Laplanche, K. Peoc'h, S. Schraen, L. Buée, F. Pasquier, J. Hugon, J. Touchon and S. Lehmann, Impact of the 2008-2012 French Alzheimer Plan on the Use of Cerebrospinal Fluid Biomarkers in Research Memory Center: The PLM Study (1) 297-305

Garwood, M., see Sillerud, L.O. (2) 349-365

Gascón, J., see Blazquez-Llorca, L. (4) 995-1013

Gawinecka, J., see Dieks, J.-K. (2) 387-397

Geda, Y.E., M. Ragossnig, L.A. Roberts, R.O. Roberts, V.S. Pankratz, T.J.H. Christianson, M.M. Mielke, J.A. Levine, B.F. Boeve, O. Sochor, E.G. Tangalos, D.S. Knopman and R.C. Petersen, Caloric Intake, Aging, and Mild Cognitive Impairment: A Population-Based Study (2) 501-507
Giorgetti, B., see Balietti, M. (4) 887-896

Gmitterova, K., see Dieks, J.-K. (2) 387-397

Goh, L.K., see Chong, M.S. (2) 399-405

Goizet, C., see Le Ber, I. (2) 485-499

Golfier, V., see Le Ber, I. (2) 485-499

González-Robles, A., see Minjarez, B. (1) 239-262

Gordon, W., see Zhao, W. (2) 417-429

Grammas, P., see Sanchez, A. (1) 281-291

Gramunt, N., see Dal-Ré, R. (1) 183-190

Guerreiro, M., see Silva, D. (3) 681-689

Guillot-Noel, L., see Le Ber, I. (2) 485-499

Gupta, V.B., see O’Bryant, S.E. (4) 841-849

Haddad, R., see Désiré, L. (2) 469-483

Hadjipavlou-Litina, D., see Pontiki, E. (1) 215-230

Hall, J., see O’Bryant, S.E. (3) 701-706

Hannequin, D., see Le Ber, I. (2) 485-499

Hansson, O., see Olsson, B. (3) 673-679

Haroutunian, V., see Zhao, W. (2) 417-429

Harrington, K., see Ellis, K.A. (4) 861-871

Hauer, K, see Zieschang, T. (1) 191-202

Havas, D., see Nyborg, A.C. (4) 957-967

Hayden, E.Y., see Mizwicki, M.T. (1) 155-170

He, P., see Li, R. (3) 589-594

Hegeman-Kleinn, I., see Nabuurs, R.J.A. (4) 1037-1049

Heidrich, J.E., see Sillerud, L.O. (2) 349-365

Heinemann, U., see Dieks, J.-K. (2) 387-397

Helmer, C., see Pimouguet, C. (1) 307-314

Henry-Watson, J., see Dugger, B.N. (2) 529-536

Hernández, I., see Espinosa, A. (3) 769-780

Hertze, J., see Olsson, B. (3) 673-679

Hewitt, K.N., see Scullion, G.A. (3) 781-793

Hidalgo, J.A., see Dugger, B.N. (2) 529-536

Higashiuesato, Y., see Katsumata, Y. (1) 273-279

Ho, L., see Zhao, W. (2) 417-429

Höglund, K., see Olsson, B. (3) 673-679

Honea, R.A., see Royall, D.R. (2) 547-560

Hu, Y., see Li, X.-H. (3) 755-767

Hugon, J., see Gabelle, A. (1) 297-305

Hutter-Paier, B., see Nyborg, A.C. (4) 957-967

Ianni, M., see Porcellini, E. (2) 439-447

Inestrosa, N.C., see Abbott, A.C. (4) 873-885

Innamorati, G., see Valenti, M.T. (1) 263-271

Izco, M., P. Pesini, V. Pérez-Grijalba, N. Fandos and M. Sarasa, Optimized Protocol for Amyloid- $\beta$ Extraction from the Brain (4) 835-839

Jacob, C., see Jonveaux, T.R. (1) 325-338

Jagt, D.L.V., see Sillerud, L.O. (2) 349-365

Jagt, T.A.V., see Sillerud, L.O. (2) 349-365 
Jin, M.-w., see Li, X.-H. (3) 755-767

Jin, Z.-d., see Li, X.-H. (3) 755-767

Johansson, B., see Rolstad, S. (4) 949-956

Johnson, K., see Perez, D.L. (1) 139-144

Johnson, L., see O’Bryant, S.E. (3) 701-706

Jonveaux, T.R., M. Batt, R. Fescharek, A. Benetos, A. Trognon, S.B. Chuzeville, A. Pop, C. Jacob, M. Yzoard, L. Demarche, L. Soulon, G. Malerba and B. Bouvel, Healing Gardens and Cognitive Behavioral Units in the Management of Alzheimer's Disease Patients: The Nancy Experience (1) 325-338

Joos, T., see Laske, C. (2) 367-375

Jothishankar, B., see Li, R. (3) 589-594

Jurick, S.M., see Wierenga, C.E. (4) 921-935

Kadish, I., see van Groen, T. (3) 609-620

Kalbe, E., P. Calabrese, S. Fengler and J. Kessler, DemTect, PANDA, EASY, and MUSIC: Cognitive Screening Tools with Age Correction and Weighting of Subtests According to Their Sensitivity and Specificity (4) 813-834

Kalinine, E., see Espinosa, J. (2) 509-518

Katsumata, Y., H. Todoriki, Y. Higashiuesato, S. Yasura, Y. Ohya, D.C. Willcox and H.H. Dodge, Very Old Adults with Better Memory Function have Higher Low-Density Lipoprotein Cholesterol Levels and Lower Triglyceride to High-Density Lipoprotein Cholesterol Ratios: KOCOA Project (1) 273-279

Kaur, G., see Choi, J.H.K. (3) 691-700

Kazlauckas, V., see Espinosa, J. (2) 509-518

Keeser, D., see Blautzik, J. (3) 741-754

Kehoe, P.G., see Dwyer, R. (1) 145-154

Kemmler, G., see Defrancesco, M. (3) 665-672

Kessler, J., see Kalbe, E. (4) 813-834

Kim, B., C. Backus, S. Oh and E.L. Feldman, Hyperglycemia-Induced Tau Cleavage in vitro and in vivo: A Possible Link Between Diabetes and Alzheimer's Disease (3) 727-739

Kim, J., see Won, J.-S. (3) 621-635

Kirsch, V., see Blautzik, J. (3) 741-754

Knable, L., see Zhao, W. (2) 417-429

Knopman, D.S., see Geda, Y.E. (2) 501-507

Kolb, H.C., see Chien, D.T. (2) 457-468

Komici, K., see Femminella, G.D. (2) 341-347

Konrad, R.J., see Lachno, D.R. (4) 897-910

Kontogiorgis, C., see Pontiki, E. (1) 215-230

Korolev, I.O., see Zhu, D.C. (4) 969-984

Kühbach, K., see Wang-Dietrich, L. (4) 985-994

Kuznicki, J., see Wojda, U. (3) 563-588

Kyratzi, E., see Chatzistavraki, M. (2) 407-415
Lachno, D.R., B.A. Evert, H. Vanderstichele, M. Robertson, R.B. DeMattos, R.J. Konrad, J.A. Talbot, M.M. Racke and R.A. Dean, Validation of Assays for Measurement of Amyloid- $\beta$ Peptides in Cerebrospinal Fluid and Plasma Specimens from Patients with Alzheimer's Disease Treated with Solanezumab (4) 897-910

Lacomblez, L., see Le Ber, I. (2) 485-499

Lannfelt, L., see Olsson, B. (3) 673-679

Laplanche, J.-L., see Gabelle, A. (1) 297-305

Laske, C., M. Schmohl, T. Leyhe, E. Stransky, W. Maetzler, D. Berg, A.J. Fallgatter, T. Joos and J. Dietzsch, Immune Profiling in Blood Identifies sTNF-R1 Performing Comparably Well as Biomarker Panels for Classification of Alzheimer's Disease Patients (2) 367-375

Lavallart, B., see Pimouguet, C. (1) 307-314

Le Ber, I., A. Camuzat, L. Guillot-Noel, D. Hannequin, L. Lacomblez, V. Golfier, M. Puel, O. Martinaud, V. Deramecourt, S. Rivaud-Pechoux, S. Millecamps, M. Vercelletto, P. Couratier, F. Sellal, F. Pasquier, F. Salachas, C. Thomas-Antérion, M. Didic, J. Pariente, D. Seilhean, M. Ruberg, I. Wargon, F. Blanc, W. Camu, B.-F. Michel, E. Berger, M. Sauvée, C. Thauvin-Robinet, K. Mondon, E. Tournier-Lasserve, C. Goizet, M. Fleury, G. Viennet, P. Verpillat, V. Meininger, C. Duyckaerts, B. Dubois, A. Brice and the French research network on FTLD/FTLD-ALS, C9ORF72 Repeat Expansions in the Frontotemporal Dementias Spectrum of Diseases: A Flow-chart for Genetic Testing (2) 485-499

Lee, T.S., see Chong, M.S. (2) 399-405

Lee, T.S., see Feng, L. (3) 649-657

Lehmann, S., see Gabelle, A. (1) 297-305

Lemaire, J.J., see Fontaine, D. (1) 315-323

Leon, A., see Cagnin, A. (2) 377-385

Leosco, D., see Femminella, G.D. (2) 341-347

Levine, J.A., see Geda, Y.E. (2) 501-507

Levy, E., see Choi, J.H.K. (3) 691-700

Leyhe, T., see Laske, C. (2) 367-375

Li, R., J. Cui, B. Jothishankar, J. Shen, P. He and Y. Shen, Early Reproductive Experiences in Females Make Differences in Cognitive Function Later in Life (3) 589-594

Li, S., see Tang, M. (1) 203-213

Li, X.-H., X. Xin, Y. Wang, J.-z. Wu, Z.-d. Jin, L.n. Ma, C.-j. Nie, X. Xiao, Y. Hu and M.-w. Jin, Pentamethylquercetin Protects Against DiabetesRelated Cognitive Deficits in Diabetic GotoKakizaki Rats (3) 755-767 
Licastro, F., see Porcellini, E. (2) 439-447

Liccardo, D., see Femminella, G.D. (2) 341-347

Lim, W.S., see Chong, M.S. (2) 399-405

Lim, W.S., see Feng, L. (3) 649-657

Lim, Y.Y., see Ellis, K.A. (4) 861-871

Liu, G., see Mizwicki, M.T. (1) 155-170

Liu, T.T., see Wierenga, C.E. (4) 921-935

Lizio, R., see Babiloni, C. (3) 707-725

Lizio, R., see Babiloni, C. (4) 1015-1035

López, O., see Espinosa, A. (3) 769-780

Luna-Arias, J.P., see Minjarez, B. (1) 239-262

Luna-Muñoz, J., see Minjarez, B. (1) 239-262

Luo, J., see Sanchez, A. (1) 281-291

Luo, Y., see Pontiki, E. (1) 215-230

Luo, Y., see Zhou, Y. (4) 937-947

Ma, L.-n., see Li, X.-H. (3) 755-767

MacLusky, N.J., see Tierney, M.C. (3) 601-608

Maetzler, W., see Laske, C. (2) 367-375

Magpantay, L., see Mizwicki, M.T. (1) 155-170

Mahanian, M., see Mizwicki, M.T. (1) 155-170

Majumdar, S., see Zhu, D.C. (4) 969-984

Malerba, G., see Jonveaux, T.R. (1) 325-338

Manara, R., see Tagliapietra, M. (1) 231-238

Mariner, M., see Dugger, B.N. (2) 529-536

Marino, S., see Babiloni, C. (4) 1015-1035

Marksteiner, J., see Defrancesco, M. (3) 665-672

Maroco, J., see Silva, D. (3) 681-689

Martelli, P.L., see Porcellini, E. (2) 439-447

Martinaud, O., see Le Ber, I. (2) 485-499

Martinez, J., see Sanchez, A. (1) 281-291

Martínez-Martín, P., see Carmona, P. (4) 911-920

Martins de Lima, M.N., see Schröder, N. (4) 797-812

Martins, R., see O’Bryant, S.E. (4) 841-849

Martins, R.N., see Ellis, K.A. (4) 861-871

Martins, R.N., see Rembach, A. (1) 171-182

Maruff, P., see Ellis, K.A. (4) 861-871

Marzano, N., see Babiloni, C. (3) 707-725

Marzano, N., see Babiloni, C. (4) 1015-1035

Massman, P., see Pavlik, V. (2) 449-456

Masters, C.L., see Ellis, K.A. (4) 861-871

Masters, C.L., see Rembach, A. (1) 171-182

Mathews, P.M., see Choi, J.H.K. (3) 691-700

Mauleón, A., see Espinosa, A. (3) 769-780

Mazzella, M.J., see Choi, J.H.K. (3) 691-700

McGinnis, S.M., see Perez, D.L. (1) 139-144

Meindl, T., see Blautzik, J. (3) 741-754

Meininger, V., see Le Ber, I. (2) 485-499

Mena, R., see Minjarez, B. (1) 239-262

Merchán-Pérez, Á., see Blazquez-Llorca, L. (4) $995-$ 1013
Merril, C.R., Is Sporadic Alzheimer's Disease Associated with Diphtheria Toxin? (3) 595-600

Michel, B.-F., see Le Ber, I. (2) 485-499

Mielke, M.M., see Geda, Y.E. (2) 501-507

Millecamps, S., see Le Ber, I. (2) 485-499

Minjarez, B., M.L.V. Rustarazo, M.M. Sanchez del Pino, A. González-Robles, J.A. Sosa-Melgarejo, J. Luna-Muñoz, R. Mena and J.P. Luna-Arias, Identification of Polypeptides in Neurofibrillary Tangles and Total Homogenates of Brains with Alzheimer's Disease by Tandem Mass Spectrometry (1) 239-262

Minthon, L., see Olsson, B. (3) 673-679

Mizwicki, M.T., G. Liu, M. Fiala, L. Magpantay, J. Sayre, A. Siani, M. Mahanian, R. Weitzman, E.Y. Hayden, M.J. Rosenthal, I. Nemere, J. Ringman and D.B. Teplow, 1 $\alpha, 25$-Dihydroxyvitamin $\mathrm{D}_{3}$ and Resolvin D1 Retune the Balance between Amyloid- $\beta$ Phagocytosis and Inflammation in Alzheimer's Disease Patients (1) 155-170

Moineddin, R., see Tierney, M.C. (3) 601-608

Molina, M., see Carmona, P. (4) 911-920

Molinuevo, J.L., see Dal-Ré, R. (1) 183-190

Moll, J.R., see Nyborg, A.C. (4) 957-967

Monaco, S., see Tagliapietra, M. (1) 231-238

Mondon, K., see Le Ber, I. (2) 485-499

Monsch, A.U., see Schmid, N.S. (2) 537-546

Montagnese, S., see Babiloni, C. (3) 707-725

Montañés, M., D. Casabona, L. Sarasa, P. Pesini and M. Sarasa, Prevention of Amyloid- $\beta$ Fibril Formation Using Antibodies Against the C-terminal Region of Amyloid- $\beta_{1-40}$ and Amyloid- $\beta_{1-42}$ (1) 133-137

Montella, P., see Babiloni, C. (4) 1015-1035

Morales-Corraliza, J., see Choi, J.H.K. (3) 691-700

Moreno-Ramos, T., J. Benito-León, A. Villarejo and F. Bermejo-Pareja, Retinal Nerve Fiber Layer Thinning in Dementia Associated with Parkinson's Disease, Dementia with Lewy Bodies, and Alzheimer's Disease (3) 659-664

Mu, F., see Chien, D.T. (2) 457-468

Mueller, S., see Blautzik, J. (3) 741-754

Muhs, A., see Froestl, W. (1) 1-114

Munafo, M., see Dwyer, R. (1) 145-154

Mundi, C., see Babiloni, C. (4) 1015-1035

Munive, V.A., see Recuero, M. (3) 637-647

Nabuurs, R.J.A., R. Natté, F.M. de Ronde, I. HegemanKleinn, J. Dijkstra, S.G. van Duinen, A.G. Webb, A.J. Rozemuller, M.A. van Buchem and L. van der Weerd, MR Microscopy of Human Amyloid- $\beta$ Deposits: Characterization of Parenchymal 
Amyloid, Diffuse Plaques, and Vascular Amyloid (4) 1037-1049

Nabuurs, R.J.A., see van Duijn, S. (4) 1051-1059

Nägga, K., see Olsson, B. (3) 673-679

Natté, R., see Nabuurs, R.J.A. (4) 1037-1049

Natté, R., see van Duijn, S. (4) 1051-1059

Nemere, I., see Mizwicki, M.T. (1) 155-170

Ng, T.P., see Chong, M.S. (2) 399-405

Ng, T.P., see Feng, L. (3) 649-657

Nie, C.-j., see Li, X.-H. (3) 755-767

Nobili, F., see Babiloni, C. (4) 1015-1035

Nunes, F., see Espinosa, J. (2) 509-518

Nyborg, A.C., J.R. Moll, R.D. Wegrzyn, D. Havas, B. Hutter-Paier, G.G.Z. Feuerstein and A.S. Rudolph, In Vivo and Ex Vivo Imaging of Amyloid- $\beta$ Cascade Aggregates with a Pronucleon ${ }^{\mathrm{TM}}$ Peptide (4) $957-967$

O’Bryant, S.E., G. Xiao, M. Edwards, M. Devous, V.B. Gupta, R. Martins, F. Zhang and R. Barber for the Texas Alzheimer's Research and Care Consortium (TARCC), Biomarkers of Alzheimer's Disease Among Mexican Americans (4) 841-849

O’Bryant, S.E., L. Johnson, M. Edwards, H. Soares, M.D. Devous, S. Ross, G. Rohlfing, J. Hall and for the Texas Alzheimer's Research \& Care Consortium, The Link Between C-Reactive Protein and Alzheimer's Disease Among Mexican Americans (3) 701-706

Oh, S., see Kim, B. (3) 727-739

Ohlsson, M., see Olsson, B. (3) 673-679

Ohya, Y., see Katsumata, Y. (1) 273-279

Olsson, B., J. Hertze, M. Ohlsson, K. Nägga, K. Höglund, H. Basun, P. Annas, L. Lannfelt, N. Andreasen, L. Minthon, H. Zetterberg, K. Blennow and O. Hansson, Cerebrospinal Fluid Levels of Heart Fatty Acid Binding Protein are Elevated Prodromally in Alzheimer's Disease and Vascular Dementia (3) 673-679

Orlando, R.A., see Sillerud, L.O. (2) 349-365

Oster, P., see Zieschang, T. (1) 191-202

Pagano, G., see Femminella, G.D. (2) 341-347

Palmer, R.F., see Royall, D.R. (2) 547-560

Pampanin, M., see Valenti, M.T. (1) 263-271

Pando, M.P., see Désiré, L. (2) 469-483

Pankratz, V.S., see Geda, Y.E. (2) 501-507

Paolini, M., see Blautzik, J. (3) 741-754

Papazafiri, P., see Chatzistavraki, M. (2) 407-415

Paquet, C., see Gabelle, A. (1) 297-305

Pardon, M.-C., see Scullion, G.A. (3) 781-793
Pariente, J., see Le Ber, I. (2) 485-499

Parisi, V., see Femminella, G.D. (2) 341-347

Pasinetti, G.M., see Zhao, W. (2) 417-429

Pasquier, F., see Le Ber, I. (2) 485-499

Pasquier, F., see Gabelle, A. (1) 297-305

Pavlik, V., P. Massman, R. Barber and R. Doody for the Texas Alzheimer's Research and Care Consortium (TARCC), Differences in the Association of Peripheral Insulin and Cognitive Function in NonDiabetic Alzheimer's Disease Cases and Normal Controls (2) 449-456

Peoc'h, K., see Gabelle, A. (1) 297-305

Percio, C.D., see Babiloni, C. (3) 707-725

Percio, C.D., see Babiloni, C. (4) 1015-1035

Perez, D.L., B.C. Dickerson, S.M. McGinnis, D. Sapolsky, K. Johnson, M. Searl and K.R. Daffner, You Don't Say: Dynamic Aphasia, Another Variant of Primary Progressive Aphasia? (1) 139-144

Pérez-Grijalba, V., see Izco, M. (4) 835-839

Pertile, K.K., see Rembach, A. (1) 171-182

Pesini, P., see Izco, M. (4) 835-839

Pesini, P., see Montañés, M. (1) 133-137

Pession, A., see Porcellini, E. (2) 439-447

Petersen, R.C., see Geda, Y.E. (2) 501-507

Pfeifer, A., see Froestl, W. (1) 1-114

Pimouguet, C., V. Bassi, D. Somme, B. Lavallart, C. Helmer and J.F. Dartigues, The 2008-2012 French Alzheimer Plan: A Unique Opportunity for Improving Integrated Care for Dementia (1) 307314

Pontiki, E., C. Kontogiorgis, Y. Xu, D. HadjipavlouLitina and Y. Luo, New Lipoxygenase Inhibitors of Reactive Oxygen Species Production in Cellular Models of Amyloid ( $\mathrm{A}_{2}$ ) Toxicities (1) 215-230

Pop, A., see Jonveaux, T.R. (1) 325-338

Porcellini, E., I. Carbone, P.L. Martelli, M. Ianni, R. Casadio, A. Pession and F. Licastro, Haplotype of Single Nucleotide Polymorphisms in Exon 6 of the MZF-1 Gene and Alzheimer's Disease (2) 439-447

Porciúncula, L.O., see Espinosa, J. (2) 509-518

Puel, M., see Le Ber, I. (2) 485-499

Qiao, Z., see Tang, M. (1) 203-213

Racke, M.M., see Lachno, D.R. (4) 897-910

Ragossnig, M., see Geda, Y.E. (2) 501-507

Rankin, S., see Tierney, M.C. (3) 601-608

Razzouk, M., see Fontaine, D. (1) 315-323

Recuero, M., V.A. Munive, I. Sastre, J. Aldudo, F. Valdivieso and M.J. Bullido, A Free Radical- 
Generating System Regulates A $\beta$ PP Metabolism/ Processing: Involvement of the Ubiquitin/ Proteasome and Autophagy/Lysosome Pathways (3) $637-647$

Rego, A.C., see Caldeira, G.L. (1) 115-131

Reiser, M., see Blautzik, J. (3) 741-754

Rembach, A., J.D. Doecke, B.R. Roberts, A.D. Watt, N.G. Faux, I. Volitakis, K.K. Pertile, R.L. Rumble, B.O. Trounson, C.J. Fowler, W. Wilson, K.A. Ellis, R.N. Martins, C.C. Rowe, V.L. Villemagne, D. Ames, C.L. Masters, AIBL research group, A.I. Bush, Longitudinal Analysis of Serum Copper and Ceruloplasmin in Alzheimer's Disease (1) 171182

Rengo, G., see Femminella, G.D. (2) 341-347

Richardson, J.C., see Babiloni, C. (4) 1015-1035

Ringman, J., see Mizwicki, M.T. (1) 155-170

Ripoche, E., see Ancelin, M.-L. (2) 519-528

Rissman, R.A., see Wierenga, C.E. (4) 921-935

Ritchie, K., see Ancelin, M.-L. (2) 519-528

Rivaud-Pechoux, S., see Le Ber, I. (2) 485-499

Robert, P., At the End of a Very Hard Day, People Find Some Reason to Believe (1) 295-296

Robert, P., see Fontaine, D. (1) 315-323

Roberts, B.R., see Rembach, A. (1) 171-182

Roberts, L.A., see Geda, Y.E. (2) 501-507

Roberts, R.O., see Geda, Y.E. (2) 501-507

Robertson, M., see Lachno, D.R. (4) 897-910

Rocha, A., see Espinosa, J. (2) 509-518

Rodrigues, A., see Silva, D. (3) 681-689

Rodriguez, G., see Babiloni, C. (4) 1015-1035

Rodríguez, J.-R., see Blazquez-Llorca, L. (4) 995-1013

Rohlfing, G., see O’Bryant, S.E. (3) 701-706

Rolstad, S., A.I. Berg, M. Bjerke, B. Johansson, H. Zetterberg and A. Wallin, Cerebrospinal Fluid Biomarkers Mirror Rate of Cognitive Decline (4) 949-956

Rosende-Roca, M., see Espinosa, A. (3) 769-780

Rosenthal, M.J., see Mizwicki, M.T. (1) 155-170

Ross, S., see O'Bryant, S.E. (3) 701-706

Rossini, P.M., see Babiloni, C. (3) 707-725

Rossini, P.M., see Babiloni, C. (4) 1015-1035

Rouaud, O., see Ancelin, M.-L. (2) 519-528

Rowe, C.C., see Ellis, K.A. (4) 861-871

Rowe, C.C., see Rembach, A. (1) 171-182

Royall, D.R., R.F. Palmer, E.D. Vidoni and R.A. Honea, The Default Mode Network may be the Key Substrate of Depressive Symptom-Related Cognitive Changes (2) 547-560

Rozemuller, A.J., see Nabuurs, R.J.A. (4) 1037-1049

Ruberg, M., see Le Ber, I. (2) 485-499
Rudolph, A.S., see Nyborg, A.C. (4) 957-967

Ruiz, A., see Espinosa, A. (3) 769-780

Rumble, R.L., see Rembach, A. (1) 171-182

Rustarazo, M.L.V., see Minjarez, B. (1) 239-262

Ryan, J., see Tierney, M.C. (3) 601-608

Salachas, F., see Le Ber, I. (2) 485-499

Salvatore, E., see Babiloni, C. (4) 1015-1035

Salvo, S.D., see Babiloni, C. (4) 1015-1035

Sanchez del Pino, M.M., see Minjarez, B. (1) 239-262

Sanchez, A., D. Tripathy, J. Luo, X. Yin, J. Martinez and P. Grammas, Neurovascular Unit and the Effects of Dosage in VEGF Toxicity: Role for Oxidative Stress and Thrombin (1) 281-291

Santana, I., see Silva, D. (3) 681-689

Sapolsky, D., see Perez, D.L. (1) 139-144

Sarasa, L., see Montañés, M. (1) 133-137

Sarasa, M., see Izco, M. (4) 835-839

Sarasa, M., see Montañés, M. (1) 133-137

Sastre, I., see Recuero, M. (3) 637-647

Sauvée, M., see Le Ber, I. (2) 485-499

Savage, G., see Ellis, K.A. (4) 861-871

Sayre, J., see Mizwicki, M.T. (1) 155-170

Schein, V., see Espinosa, J. (2) 509-518

Schiff, S., see Babiloni, C. (3) 707-725

Schmid, N.S., K.I. Taylor, N.S. Foldi, M. Berres and A.U. Monsch, Neuropsychological Signs of Alzheimer's Disease 8 Years Prior to Diagnosis (2) $537-546$

Schmohl, M., see Laske, C. (2) 367-375

Schocke, M., see Defrancesco, M. (3) 665-672

Schott, J.M., see Barnes, J. (2) 431-437

Schraen, S., see Gabelle, A. (1) 297-305

Schröder, N., L.S. Figueiredo and M.N. Martins de Lima, Role of Brain Iron Accumulation in Cognitive Dysfunction: Evidence from Animal Models and Human Studies (4) 797-812

Schwenk, M., see Zieschang, T. (1) 191-202

Scullion, G.A., K.N. Hewitt and M.-C. Pardon, Corticotropin-Releasing Factor Receptor 1 Activation During Exposure to Novelty Stress Protects Against Alzheimer's Disease-Like Cognitive Decline in A $\beta P P / P S 1$ Mice (3) 781-793

Searl, M., see Perez, D.L. (1) 139-144

Seilhean, D., see Le Ber, I. (2) 485-499

Sellal, F., see Le Ber, I. (2) 485-499

Serviddio, G., see Babiloni, C. (3) 707-725

Shankle, W.R., see Chien, D.T. (2) 457-468

Shen, J., see Li, R. (3) 589-594

Shen, Y., see see Li, R. (3) 589-594

Shin, D.D., see Wierenga, C.E. (4) 921-935 
Shunmugavel, A., see Won, J.-S. (3) 621-635

Siani, A., see Mizwicki, M.T. (1) 155-170

Sillerud, L.O., N.O. Solberg, R. Chamberlain, R.A. Orlando, J.E. Heidrich, D.C. Brown, C.I. Brady, T.A.V. Jagt, M. Garwood and D.L.V. Jagt, SPION-Enhanced Magnetic Resonance Imaging of Alzheimer's Disease Plaques in A $\beta$ PP/PS-1 Transgenic Mouse Brain (2) 349-365

Silva, D., M. Guerreiro, I. Santana, A. Rodrigues, S. Cardoso, J. Maroco and A. de Mendonça, Prediction of Long-Term (5 Years) Conversion to Dementia Using Neuropsychological Tests in a Memory Clinic Setting (3) 681-689

Singh, A.K., see Won, J.-S. (3) 621-635

Singh, I., see Won, J.-S. (3) 621-635

Skrobot, O.A., see Dwyer, R. (1) 145-154

Soares, H., see O’Bryant, S.E. (3) 701-706

Sochor, O., see Geda, Y.E. (2) 501-507

Sol, O., see Désiré, L. (2) 469-483

Solazzi, M., see Balietti, M. (4) 887-896

Solberg, N.O., see Sillerud, L.O. (2) 349-365

Somme, D., see Pimouguet, C. (1) 307-314

Soricelli, A., see Babiloni, C. (3) 707-725

Soricelli, A., see Babiloni, C. (4) 1015-1035

Sosa-Melgarejo, J.A., see Minjarez, B. (1) 239-262

Soulon, L., see Jonveaux, T.R. (1) 325-338

Souza, D.O., see Espinosa, J. (2) 509-518

Stecca, A., see Cagnin, A. (2) 377-385

Stern, Y., see Zahodne, L.B. (4) 851-860

Stransky, E., see Laske, C. (2) 367-375

Streich, J.-H., see Dieks, J.-K. (2) 387-397

$\mathrm{Su}, \mathrm{M}$.-Y., see Chien, D.T. (2) 457-468

Sue, L.I., see Dugger, B.N. (2) 529-536

Szardenings, A.K., see Chien, D.T. (2) 457-468

Szoeke, C., see Ellis, K.A. (4) 861-871

Tagliapietra, M., G. Zanusso, M. Fiorini, N. Bonetto, G. Zarantonello, A. Zambon, M. Ermani, S. Monaco, R. Manara and A. Cagnin, Accuracy of Diagnostic Criteria for Sporadic Creutzfeldt-Jakob Disease Among Rapidly Progressive Dementia (1) 231238

Talbot, J.A., see Lachno, D.R. (4) 897-910

Tamagnini, F., see Balietti, M. (4) 887-896

Tan, C.H., see Chong, M.S. (2) 399-405

Tang, M., Z. Wang, Y. Zhou, W. Xu, S. Li, L. Wang, D. Wei and Z. Qiao, A Novel Drug Candidate for Alzheimer's Disease Treatment: gx-50 Derived from Zanthoxylum Bungeanum (1) 203-213

Tangalos, E.G., see Geda, Y.E. (2) 501-507

Tárraga, L., see Espinosa, A. (3) 769-780
Tay, L., see Chong, M.S. (2) 399-405

Taylor, K.I., see Schmid, N.S. (2) 537-546

Tedeschi, G., see Babiloni, C. (4) 1015-1035

Teipel, S.J., see Blautzik, J. (3) 741-754

Teplow, D.B., see Mizwicki, M.T. (1) 155-170

Thauvin-Robinet, C., see Le Ber, I. (2) 485-499

Thomas-Antérion, C., see Le Ber, I. (2) 485-499

Tierney, M.C., J. Ryan, M.-L. Ancelin, R. Moineddin, S. Rankin, C. Yao and N.J. MacLusky, Lifelong Estrogen Exposure and Memory in Older Postmenopausal Women (3) 601-608

Todoriki, H., see Katsumata, Y. (1) 273-279

Toledano, A., see Carmona, P. (4) 911-920

Toledo, C.C., see Abbott, A.C. (4) 873-885

Touchon, J., see Gabelle, A. (1) 297-305

Tournier-Lasserve, E., see Le Ber, I. (2) 485-499

Tripathy, D., see Sanchez, A. (1) 281-291

Trognon, A., see Jonveaux, T.R. (1) 325-338

Trounson, B.O., see Rembach, A. (1) 171-182

Ursini, F., see Babiloni, C. (4) 1015-1035

Valdivieso, F., see Recuero, M. (3) 637-647

Valenti, M.T., S. Bolognin, C. Zanatta, L. Donatelli, G. Innamorati, M. Pampanin, G. Zanusso, P. Zatta and L.D. Carbonare, Increased Glutaminyl Cyclase Expression in Peripheral Blood of Alzheimer's Disease Patients (1) 263-271

Valero, S., see Espinosa, A. (3) 769-780

van Buchem, M.A., see Nabuurs, R.J.A. (4) 1037-1049 van Buchem, M.A., see van Duijn, S. (4) 1051-1059 van der Weerd, L., see Nabuurs, R.J.A. (4) 1037-1049 van Duijn, S., R.J.A. Nabuurs, S.G. van Duinen, R. Natté, M.A. van Buchem and A. Alia, Longitudinal Monitoring of Sex-Related in vivo Metabolic Changes in the Brain of Alzheimer's Disease Transgenic Mouse Using Magnetic Resonance Spectroscopy (4) 1051-1059

van Duinen, S.G., see Nabuurs, R.J.A. (4) 1037-1049

van Duinen, S.G., see van Duijn, S. (4) 1051-1059

van Groen, T., I. Kadish, S.A. Funke, D. Bartnik and D. Willbold, Treatment with D3 Removes Amyloid Deposits, Reduces Inflammation, and Improves Cognition in Aged A $\beta$ PP/PS1 Double Transgenic Mice (3) 609-620

Vanderstichele, H., see Lachno, D.R. (4) 897-910

Varela-Nallar L., see Abbott, A.C. (4) 873-885

Varges, D., see Dieks, J.-K. (2) 387-397

Varghese, M., see Zhao, W. (2) 417-429

Vecchio, F., see Babiloni, C. (3) 707-725

Vercelletto, M., see Le Ber, I. (2) 485-499 
Vercruysse, O., see Gabelle, A. (1) 297-305

Vernieri, F., see Babiloni, C. (4) 1015-1035

Verpillat, P., see Le Ber, I. (2) 485-499

Vianello, D., see Cagnin, A. (2) 377-385

Viau, P., see Fontaine, D. (1) 315-323

Vidoni, E.D., see Royall, D.R. (2) 547-560

Viennet, G., see Le Ber, I. (2) 485-499

Vigorito, C., see Femminella, G.D. (2) 341-347

Villarejo, A., see Moreno-Ramos, T. (3) 659-664

Villemagne, V.L., see Ellis, K.A. (4) 861-871

Villemagne, V.L., see Rembach, A. (1) 171-182

Vinyes-Junqué, G., see Espinosa, A. (3) 769-780

Volitakis, I., see Rembach, A. (1) 171-182

Wallin, A., see Rolstad, S. (4) 949-956

Walsh, J.C., see Chien, D.T. (2) 457-468

Wang, K., see Wang-Dietrich, L. (4) 985-994

Wang, L., see Tang, M. (1) 203-213

Wang, Y., see Li, X.-H. (3) 755-767

Wang, Z., see Tang, M. (1) 203-213

Wang-Dietrich, L., S.A. Funke, K. Kühbach, K. Wang, A. Besmehn, S. Willbold, Y. Cinar, O. Bannach, E. Birkmann and D. Willbold, The Amyloid- $\beta$ Oligomer Count in Cerebrospinal Fluid is a Biomarker for Alzheimer's Disease (4) 985-994

Wargon, I., see Le Ber, I. (2) 485-499

Watt, A.D., see Rembach, A. (1) 171-182

Webb, A.G., see Nabuurs, R.J.A. (4) 1037-1049

Wegrzyn, R.D., see Nyborg, A.C. (4) 957-967

Wei, D., see Tang, M. (1) 203-213

Weitzman, R., see Mizwicki, M.T. (1) 155-170

Wierenga, C.E., L.R. Clark, S.I. Dev, D.D. Shin, S.M. Jurick, R.A. Rissman, T.T. Liu and M.W. Bondi, Interaction of Age and APOE Genotype on Cerebral Blood Flow at Rest (4) 921-935

Willbold, D., see van Groen, T. (3) 609-620

Willbold, D., see Wang-Dietrich, L. (4) 985-994

Willbold, S., see Wang-Dietrich, L. (4) 985-994

Willcox, D.C., see Katsumata, Y. (1) 273-279

Wilson, W., see Rembach, A. (1) 171-182

Wojda, U. and J. Kuznicki, Alzheimer's Disease Modeling: Ups, Downs, and Perspectives for Human Induced Pluripotent Stem Cells (3) 563588

Won, J.-S., J. Kim, B. Annamalai, A. Shunmugavel, I. Singh and A.K. Singh, Protective Role of S-Nitrosoglutathione (GSNO) Against Cognitive Impairment in Rat Model of Chronic Cerebral Hypoperfusion (3) 621-635

Wu, J.-Z., see Li, X.-H. (3) 755-767
Xiao, G., see O’Bryant, S.E. (4) 841-849

Xiao, X., see Li, X.-H. (3) 755-767

Xin, X., see Li, X.-H. (3) 755-767

Xu, W., see Tang, M. (1) 203-213

Xu, Y., see Pontiki, E. (1) 215-230

Yao, C., see Tierney, M.C. (3) 601-608

Yap, P., see Feng, L. (3) 649-657

Yasura, S., see Katsumata, Y. (1) 273-279

Yemul, S., see Zhao, W. (2) 417-429

Yin, X., see Sanchez, A. (1) 281-291

Yzoard, M., see Jonveaux, T.R. (1) 325-338

Zahodne, L.B., D.P. Devanand and Y. Stern, Coupled Cognitive and Functional Change in Alzheimer's Disease and the Influence of Depressive Symptoms (4) $851-860$

Zambon, A., see Cagnin, A. (2) 377-385

Zambon, A., see Tagliapietra, M. (1) 231-238

Zanatta, C., see Valenti, M.T. (1) 263-271

Zanusso, G., see Tagliapietra, M. (1) 231-238

Zanusso, G., see Valenti, M.T. (1) 263-271

Zarantonello, G., see Cagnin, A. (2) 377-385

Zarantonello, G., see Tagliapietra, M. (1) 231-238

Zatta, P., see Valenti, M.T. (1) 263-271

Zerr, I., see Dieks, J.-K. (2) 387-397

Zetterberg, H., see Olsson, B. (3) 673-679

Zetterberg, H., see Rolstad, S. (4) 949-956

Zhang, F., see O'Bryant, S.E. (4) 841-849

Zhao, W., L. Ho, M. Varghese, S. Yemul, K. DamsO'Connor, W. Gordon, L. Knable, D. Freire, V. Haroutunian and G.M. Pasinetti, Decreased Level of Olfactory Receptors in Blood Cells Following Traumatic Brain Injury and Potential Association with Tauopathy (2) 417-429

Zhou, W., see Désiré, L. (2) 469-483

Zhou, Y., see Tang, M. (1) 203-213

Zhou, Y., Y. Luo and J. Dai, Axonal and Dendritic Changes are Associated with Diabetic Encephalopathy in Rats: An Important Risk Factor for Alzheimer's Disease (4) 937-947

Zhu, D.C., S. Majumdar, I.O. Korolev, K.L. Berger and A.C. Bozoki, Alzheimer's Disease and Amnestic Mild Cognitive Impairment Weaken Connections Within the Default-Mode Network: A MultiModal Imaging Study (4) 969-984

Zieschang, T., M. Schwenk, P. Oster and K. Hauer, Sustainability of Motor Training Effects in Older People with Dementia (1) 191-202 